\title{
Efectividad del equipo de respuesta rápida en el Hospital Ángeles Pedregal
}

\author{
Pedro Alejandro Elguea Echavarría, ${ }^{1}$ Christopher Hernández Cortés, ${ }^{2}$ \\ Laura Ramírez Ramírez, ${ }^{3}$ Neftalí García Gómez, ${ }^{4}$ Claudia López López, ${ }^{1}$ \\ Brenda Gómez Gómez, ${ }^{5}$ Alan Ernesto García García, ${ }^{2}$ Juan Gerardo Esponda Prado ${ }^{6}$
}

\begin{abstract}
Resumen
El equipo de respuesta rápida (ERR) tiene la función de acudir a la valoración del paciente hospitalizado que presenta datos de deterioro clínico, lo anterior con la finalidad de identificar de manera temprana al paciente grave y prevenir eventos adversos disminuyendo así la morbimortalidad hospitalaria. El objetivo del presente artículo es evaluar la efectividad del ERR en el Hospital Ángeles Pedregal. Para esto se realizó un estudio de tipo observacional, retrospectivo y transversal durante el periodo del 01 de febrero del 2014 al 30 de junio de 2015. Se analizó la supervivencia de los pacientes admitidos en la Unidad de Cuidados Intensivos comparando los que fueron valorados por el ERR con los que no tuvieron esa valoración, identificando mayor supervivencia en los pacientes que recibieron atención por el ERR (37.7 versus $21.2 \%$ respectivamente), independientemente de su gravedad. De esta manera concluimos que el ERR trabaja con efectividad logrando el reconocimiento temprano del paciente con deterioro clínico, logrando una admisión oportuna a la Unidad de Terapia Intensiva (UTI) y una mayor supervivencia.
\end{abstract}

Palabras clave: Atención rápida en hospital, urgencia hospitalaria.

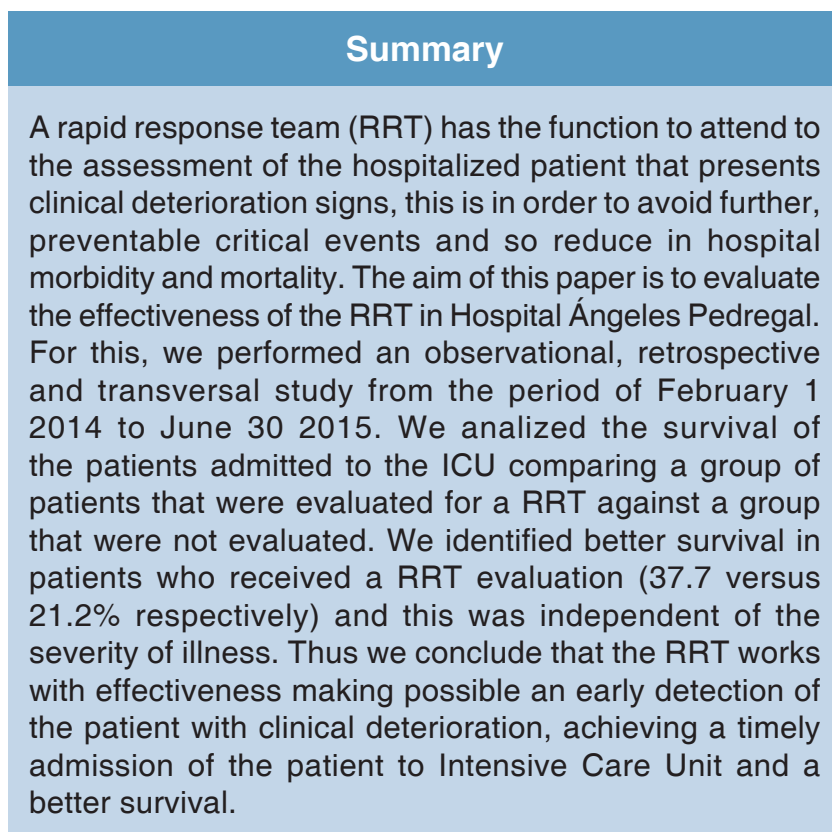

Key words: Rapid response team, hospital emergence.

\footnotetext{
${ }^{1}$ Residente del segundo año de la Especialidad en Medicina del Enfermo Adulto en Estado Crítico.

2 Residente del primer año de la Especialidad en Medicina del Enfermo Adulto en Estado Crítico.

${ }^{3}$ Maestría en Gestión y Administración de los Servicios de Salud. Coordinadora de la base de datos.

${ }^{4}$ Médico Especialista en Medicina Crítica. Maestría en Gestión y Administración de los servicios de salud.

${ }^{5}$ Residente del tercer año de la Especialidad en Medicina Interna.

${ }^{6}$ Jefe de la Unidad de Cuidados Intensivos. Profesor titular de la especialidad en Medicina del Enfermo Adulto en Estado Crítico.
}

Correspondencia:

Pedro Alejandro Elguea Echavarría

Hospital Ángeles Pedregal

Facultad Mexicana de Medicina, Universidad la Salle

Correo electrónico: alejandro_elguea@hotmail.com

Aceptado: 20-12-2016.

Este artículo puede ser consultado en versión completa en http://www.medigraphic.com/actamedica 


\section{INTRODUCCIÓN}

En ocasiones, la muerte es parte del proceso natural de la enfermedad; en otras, se debe a cuidados deficientes, manifestándose sutilmente con signos o síntomas que no se detectan y que sólo son evidentes cuando ya es demasiado tarde para revertirlos. El hecho de tener al paciente hospitalizado no garantiza la seguridad del enfermo en fase aguda. La falta de reconocimiento o respuesta al deterioro clínico en el hospital se denomina "falla para rescatar"; esto atenta contra la vida de los pacientes. ${ }^{1}$

Desde la década de los noventa se ha postulado que muchas de las muertes hospitalarias son potencialmente predecibles y prevenibles. ${ }^{2}$ Según estudios, los pacientes que cursan con deterioro clínico muestran datos objetivos y subjetivos durante las primeras seis a 24 horas antes de presentar una complicación grave. ${ }^{3}$ Un estudio mostró que $60 \%$ de eventos adversos (muerte, paro cardiorrespiratorio e ingreso no planeado a Unidad de Terapia Intensiva) presentaron anormalidades fisiológicas documentadas pero no tomadas en cuenta. ${ }^{4} \mathrm{EI}$ retraso en el tratamiento o el cuidado inadecuado de pacientes en el piso de hospitalización frecuentemente resulta en admisiones urgentes a la Unidad de Cuidados Intensivos, aumento de la estancia intrahospitalaria, paro cardiorrespiratorio y muerte. Se calcula que más de $50 \%$ de los pacientes ingresados a Unidad de Terapia Intensiva (UTI) desde la hospitalización recibieron cuidados inadecuados; así mismo, más de $41 \%$ de los ingresos fueron potencialmente evitables. ${ }^{5}$

Lamentablemente, los primeros indicadores de deterioro clínico pueden ser difíciles de identificar. Algunas de las razones son: retraso en buscar algún dato, menospreciar los datos clínicos objetivos, supervisión inadecuada, problemas organizacionales dentro del ámbito hospitalario, errores al reconocer clínicamente una urgencia, falta de entrenamiento en personal de enfermería, internos de pregrado y médicos residentes sin los conocimientos adecuados. Es de gran relevancia reforzar las bases del conocimiento en la atención del paciente crítico y del momento clave para solicitar apoyo al personal especialista en medicina crítica. ${ }^{6}$

El reconocimiento temprano del deterioro y una intervención oportuna pueden disminuir la incidencia del paro cardiaco o la necesidad de cuidados intensivos; además puede mejorar la evolución del paciente y disminuir los días de estancia hospitalaria con la ventaja administrativa de la disminución de costos. Es aquí donde entra el concepto de equipo de respuesta rápida (ERR), el cual es un algoritmo o plan de acción basado en observaciones al pie de la cama; dichas observaciones han sido recomendadas para identificar a los pacientes que presentan en algún momento datos de riesgo en las diferentes salas de hospitalización, de tal forma que puedan ser identificados y tratados de manera temprana. ${ }^{7}$

Hay cierta controversia respecto a la efectividad en la disminución de la mortalidad con la implementación del ERR y esto ha sido analizado en varias publicaciones. En un metaanálisis que incluyó ensayos clínicos desarrollados desde enero de 1990 a diciembre de 2013 usando PubMed, CINAHK y Cochrane se llegó a la conclusión de que los equipos de respuesta rápida se asocian a una disminución en la mortalidad hospitalaria y en la incidencia de paro cardiorrespiratorio. Además menciona que no es un factor determinante la presencia de un médico dentro del personal del ERR para lograr la disminución de la mortalidad. ${ }^{8}$

Para valorar la efectividad del ERR, el Comité de Enlace Internacional en Resucitación (ILCOR) realizó en el año 2007 una lista de criterios centrales y suplementarios estilo Utstein para monitorizar la incidencia y los resultados de las emergencias hospitalarias y la intervención del ERR. ${ }^{9}$ Asimismo, el Instituto por Mejoría en la Atención de la Salud (IHI por sus siglas en inglés) recomienda medir la efectividad mediante los siguientes indicadores: códigos por 1,000 altas hospitalarias (se denomina código a la necesidad de intubación endotraqueal inmediata), número de códigos fuera de la UTI y utilización del equipo de respuesta rápida (número de llamadas al equipo de respuesta rápida). ${ }^{10}$

Se han desarrollado algunos otros marcadores que pueden ser utilizados para medir la efectividad del ERR. Uno de ellos es el tiempo puerta-terapia, el cual determina el tiempo transcurrido a partir del establecimiento de deterioro clínico hasta el ingreso del paciente a la UTI. Existe un estudio realizado en Reino Unido donde se determinó una diferencia significativa en la gravedad de los pacientes (medida por APACHE II), siendo ésta mayor cuando el tiempo puerta-terapia sobrepasa las cuatro horas. De esta manera, si el paciente tarda más de este tiempo en ingresar a la UTI se interpreta como falla en la detección del paciente grave, retraso en la atención o problemas logísticos que repercuten en el pronóstico del paciente. ${ }^{11}$ Además, es importante considerar si los pacientes ingresados a la UTI fueron valorados por el ERR.

Por otro lado, es importante analizar las muertes ocurridas en el hospital. Determinar si se trató de una muerte esperada, cuidados paliativos o si el paciente presentó datos clínicos de deterioro previos a sufrir un paro cardiorrespiratorio, ya que en este caso se tratará de una "falla para rescatar". Un análisis de 576 defunciones reportadas en la Agencia Nacional para la Seguridad del Paciente en el Reino Unido en el año 2005 identificó que $11 \%(n=66)$ fueron resultado de deterioro clínico no identificado. ${ }^{12}$ 
Otra herramienta más que puede ser utilizada es estimar la gravedad de los pacientes atendidos por el ERR mediante un predictor de mortalidad. De esta manera, analizar los datos y realizar la calibración de mortalidad esperada versus mortalidad real permite medir la efectividad de la atención del paciente crítico. Existen distintos modelos dentro de los cuales podemos mencionar: APACHE I, II y III; SAPS 1, 2 y 3, MPM I y II. ${ }^{13}$ En México se realizó un estudio comparativo entre SAPS 3 y APACHE II en el que se analizó estadísticamente su capacidad discriminativa y calibración, determinando una mejor correlación del predictor de mortalidad SAPS 3 para la población mexicana. ${ }^{14}$ Asimismo, otra herramienta útil es el SOFA score, el cual determina la gravedad del paciente valorando la disfunción o fallas orgánicas y estableciendo una probabilidad de morir a partir de estos parámetros. ${ }^{15}$ Cabe mencionar que los modelos citados previamente se utilizan especialmente en los pacientes ingresados a la UTI; de esta manera, es necesario contar con una escala que permita calificar la gravedad de los pacientes en el área de hospitalización. Por este motivo se desarrolló la escala de advertencia temprana (Early Warning Score), la cual ha sido validada para su aplicación e incluso como criterio para activar el equipo de respuesta rápida en el hospital. ${ }^{16}$

Desde febrero del año 2014 se implementó en el Hospital Ángeles Pedregal el sistema de ERR. Para esto se establecieron datos de deterioro clínico (Cuadro 1). En el momento en que se detecta a un paciente que presente alguno de estos puntos se procede a solicitar por vía telefónica al conmutador el voceo "Código de respuesta rápida" indicando el número de habitación en el que se requiere del apoyo. El llamado se escucha solamente dentro de la UTI para que en ese momento un médico especialista en cuidados críticos, en compañía de una enfermera intensivista y un residente de la misma especialidad, acudan a valorar al paciente. Tras realizar un interrogatorio y exploración física podrán decidir intervenciones críticas o, de ser necesario, el ingreso del paciente a la UTI. El sistema está disponible las 24 horas del día, los 365 días del año.

El objetivo del presente trabajo es evaluar la efectividad del trabajo del equipo de respuesta rápida en el Hospital Ángeles Pedregal tomando en cuenta el impacto en la supervivencia y el tiempo de ingreso a UTI.

\section{MATERIAL Y MÉTODOS}

Se realizó un estudio de tipo observacional, retrospectivo, transversal, descriptivo y comparativo (revisión de casos), durante el periodo comprendido entre el 01 de febrero de 2014 hasta el 30 de junio de 2015 en el Hospital Ángeles Pedregal.

Para el estudio se incluyeron pacientes hospitalizados de ambos géneros de entre 18 y 95 años de edad y posteriormente ingresados en la UTI.

No se incluyeron pacientes del Área de Ginecología, Pediatría, Neonatología, Urgencias o que provenían del Área de Quirófano o Hemodinamia. Tampoco pacientes en quienes se consideró limitar el esfuerzo terapéutico.

Para la estandarización de gravedad se utilizó el predictor de mortalidad SAPS 3 y SOFA score, los cuales se aplican al momento del ingreso a UTI. Además, se calificó de manera retrospectiva el NEWS score (National Early Warning Score), el cual califica la gravedad de los pacientes que se encuentran en el área de hospitalización brindando cierto puntaje con base en los signos vitales. Se recomienda Ilamar al ERR para valorar el ingreso del paciente a la UTI cuando el puntaje es mayor o igual a 7 puntos o alguna categoría se encuentra en 3 (Cuadro 2).

Los pacientes ingresados a UTI se clasificaron en dos grupos: "Valorados por ERR" y "No valorados por ERR", denominándolos como grupo 1 y grupo 2 respectivamente.

Cuadro 1. Criterios de activación para el equipo de respuesta rápida en el Hospital Ángeles Pedregal.

1. Impresión de la gravedad

2. Dificultad respiratoria

3. Saturación de oxígeno

4. Frecuencia respiratoria

5. Frecuencia cardiaca

6. Tensión arterial sistólica

7. Gasto urinario

8. Cambio agudo del estado de alerta Confusión/desorientación

9. Hemorragia

10. Dolor torácico

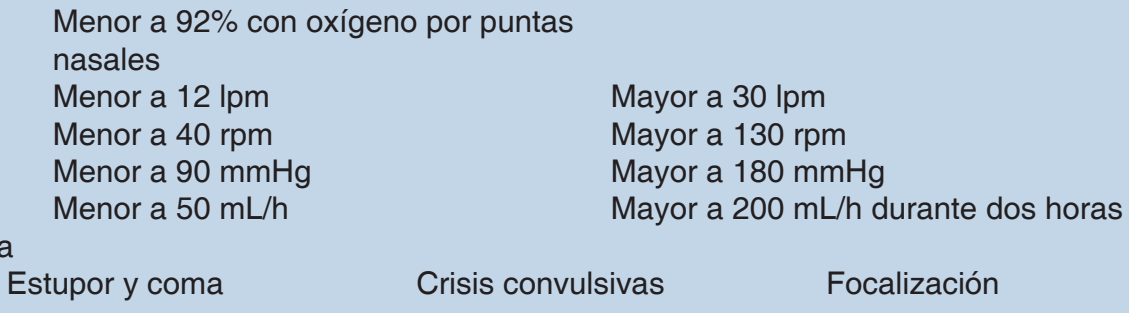




\begin{tabular}{|c|c|c|c|c|c|c|c|}
\hline \multicolumn{8}{|c|}{ Cuadro 2. National Early Warning Score. ${ }^{16}$} \\
\hline Parámetros fisiológicos & 3 & 2 & 1 & 0 & 1 & 2 & 3 \\
\hline Frecuencia respiratoria & $\leq 8$ & & $9-11$ & $12-20$ & & $21-24$ & $\geq 25$ \\
\hline Saturación de oxígeno & $\leq 91$ & $92-93$ & $94-95$ & $\geq 96$ & & & \\
\hline Oxígeno suplementario & & Sí & & No & & & \\
\hline Temperatura & $\leq 35.0$ & & $35.1-36.0$ & $36.1-38.0$ & $38.1-39.0$ & $\geq 39.1$ & \\
\hline Tensión arterial sistólica & $\leq 90$ & $91-100$ & $101-110$ & $111-219$ & & & $\geq 220$ \\
\hline Frecuencia cardiaca & $\leq 40$ & & $41-50$ & $51-90$ & $91-110$ & $111-130$ & $\geq 131$ \\
\hline Nivel de consciencia & & & & A & & & $\overline{\mathrm{V}}, \mathrm{D}, \mathrm{I}$ \\
\hline
\end{tabular}

Se revisaron los expedientes para recabar datos demográficos y clínicos, determinar su gravedad y el momento en que sufrieron deterioro clínico, así como la forma en que egresaron del hospital.

Por otro lado, se solicitó al archivo clínico del hospital el expediente de los pacientes fallecidos durante el 2014 en el Área de Hospitalización para así analizar, con base en las hojas de enfermería y notas médicas de evolución, si tuvieron datos clínicos de deterioro horas previas a su fallecimiento. Así se puede determinar si el paciente pudo ser candidato a UTI o si se trató de una muerte esperada que se encontraba en cuidados paliativos.

Posteriormente se clasificó la información y se registraron las variables mencionadas de acuerdo al diseño metodológico que se encuentra en la figura 1.

Los datos recabados en la base de datos se ingresaron al programa SPSS versión 21. Se realizó el análisis estadístico descriptivo de los datos demográficos clasificando las variables; se obtuvieron medidas de tendencia central y no central según se requiriera. Posteriormente se realizó el análisis comparativo utilizando tablas de contingencia para cruzar las variables de interés entre el grupo 1 y el grupo 2. Y con objeto de determinar la efectividad del ERR, se realizaron medidas de asociación estadística entre las variables utilizando como pruebas estadísticas para comprobar la asociación de las variables y el contraste de las hipótesis (Kolmogorov-Smirnov, binomial, $\mathrm{p}<0.05$; chicuadrada $\left(\chi^{2}\right) p<0.05$, y cuando se requirió comparar dos muestras independientes o con distribuciones anormales se realizó Kruskal-Wallis $\mathrm{p}<0.05)$. Se procedió a generar gráficos.

\section{RESULTADOS}

\section{Frecuencia de activación del ERR}

Durante el periodo comprendido de febrero de 2014 a junio de 2015 se realizaron 142 valoraciones por el Equipo de Respuesta Rápida (ERR). Setenta (49.3\%) de estas valoraciones se realizaron en el año de 2014 y 72 (50.7\%) en 2015. Lo anterior se representa en la figura 2.

\section{Pacientes fallecidos en el Área de Hospitalización}

En el Área de Hospitalización en el periodo evaluado faIlecieron 66 pacientes de un total de 6,897 hospitalizados. 61 defunciones se trataron de pacientes en quienes se limitó el esfuerzo terapéutico debido a una enfermedad terminal o irreversible.

De los cinco pacientes restantes, una defunción correspondió a muerte súbita: la paciente tenía signos vitales normales previo a presentar un paro cardiorrespiratorio. Los cuatro casos restantes se consideraron "falla para rescatar", ya que presentaron datos clínicos de deterioro previo a su fallecimiento y no se activó el equipo de respuesta rápida para su valoración.

\section{Pacientes ingresados a la Unidad de Terapia Intensiva}

El universo total estudiado del 01 de febrero de 2014 al 30 de junio de 2015 en el Hospital Ángeles Pedregal fue de 191 pacientes.

\section{Datos demográficos}

De los pacientes ingresados a la UTI encontramos que la media de edad fue $66.88 \pm 14$ con una moda de 59 (9.3\%). Fueron 109 mujeres (57.1\%) y 82 hombres estudiados (42.9\%). De acuerdo al tipo de enfermedad, el $60.7 \%$ (116) resultaron padecimientos metabólicos, 25\% (48) quirúrgicos, 1.6\% (3) traumatológicos, 3.7\% (7) cardiológicos y el $8.9 \%$ (17) oncológicos. Dentro de las comorbilidades más frecuentes en el grupo estudiado, el 45.5\% (80) con Hipertensión Arterial Sistémica, 19.9\% (38) con diabetes mellitus, $7.3 \%$ (14) con enfermedad pulmonar obstructiva crónica y el $13.1 \%$ (25) con enfermedad renal crónica. Los motivos de ingreso más frecuentes fueron: insuficiencia respiratoria en el $28.3 \%$ (54), sepsis abdominal en $3.7 \%$ (7), sangrado de tubo 


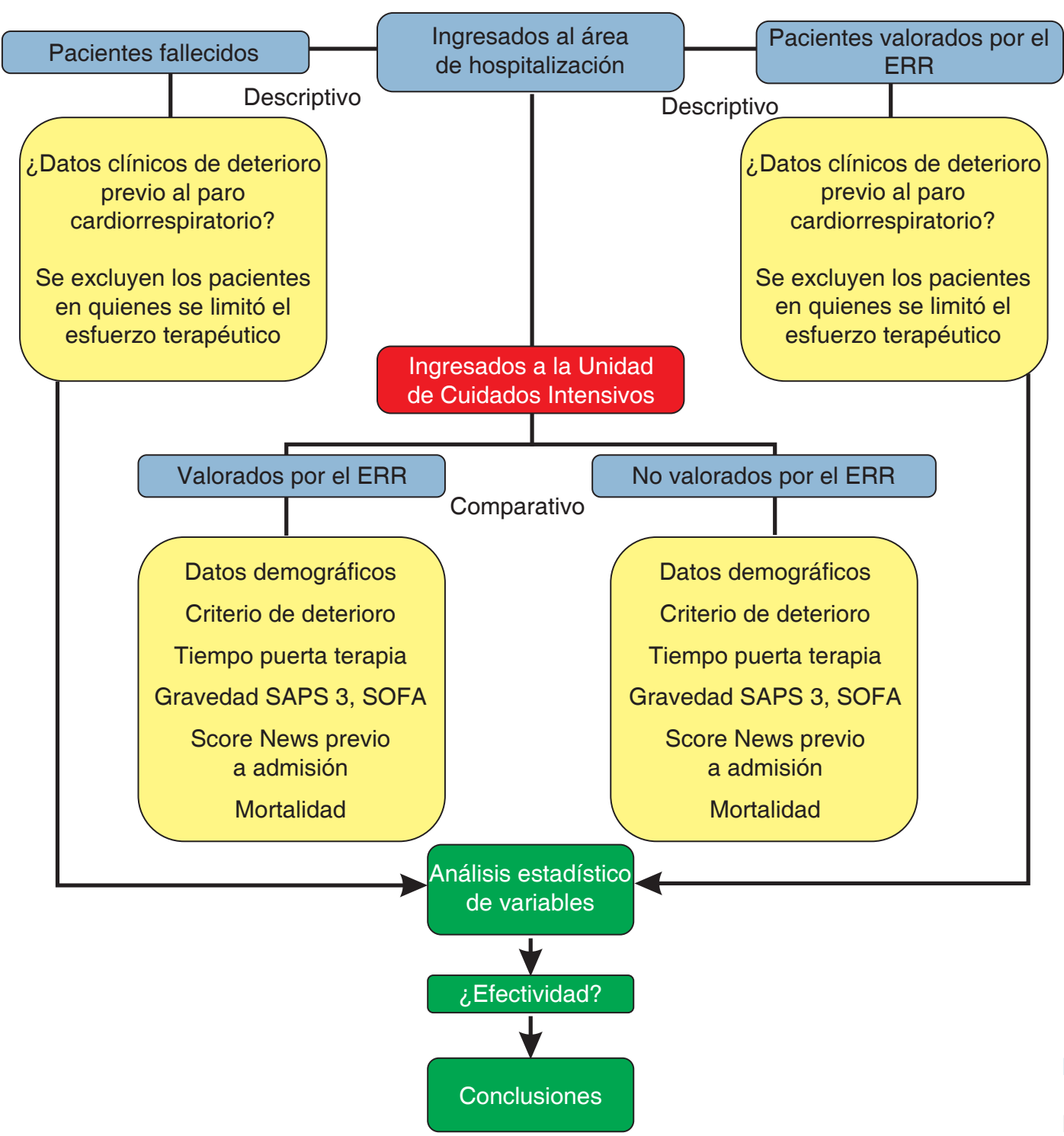

Figura 1.

Diseño metodológico.

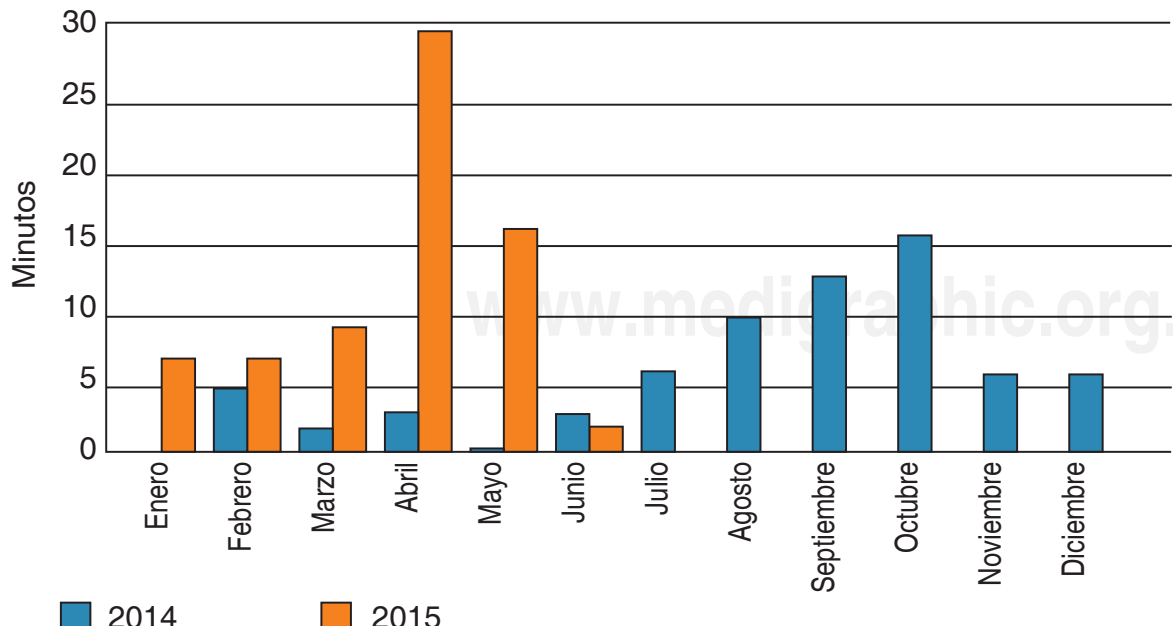

Figura 2.

Frecuencia en tiempo del uso del equipo de respuesta rápida. 
digestivo en $3.7 \%$ (7), crisis convulsivas en $2.6 \%$ (5) y arritmia cardiaca en $2.6 \%$ (5).

\section{Gravedad}

Con respecto a la variable NEWS (rango tomado para la gravedad) obtuvimos una mediana de 8 puntos.

De acuerdo al valor pronóstico de SAPS 3 se obtuvo una mediana de $20 \%$ de probabilidad de muerte, mientras que en el SOFA score se registró una mediana de 5 puntos.

\section{Días de estancia}

Encontramos una mediana de 6.4 días y una moda de 1 día que corresponde al $24.6 \%$ (47 pacientes). Como dato importante, el 14.1\% (28 pacientes) permaneció más de 10 DIAS en UTI.

\section{Forma de egreso}

El 72.3\% (138) de los pacientes vivió, el 19.4\% (37) falleció el $7.3 \%$ (14) fue trasladado a otro hospital y el 1\% (2) egresó como RCP2.

Pacientes valorados por el equipo de respuesta rápida (ERR) en contraste con los no valorados por el ERR

Se clasificó a los pacientes en dos grupos: valorados por el ERR (grupo 1), en total 152 con un porcentaje de $79.6 \%$, y los NO valorados por el ERR (grupo 2) 39, con un porcentaje de $20.4 \%$.

Ya clasificados los grupos en 1 y 2 respectivamente se analizaron las siguientes variables:

\section{Tiempo puerta UTI}

De los pacientes valorados por el equipo de respuesta rápida (ERR) el tiempo PUERTA UTI fue menor a 4 horas $72.2 \%(n=110)$, a diferencia de los que no fueron valorados por el equipo de respuesta rápida en un $45.6 \%(n=$ 17), obteniendo una $p=0.00$ con la prueba de KruskalWallis (Figura 3).

\section{Supervivencia de los pacientes que requirieron intubación inmediata}

Los pacientes que requirieron intubación inmediata representaron $54.5 \%$ de la muestra total. De estos pacientes valorados por el equipo de respuesta rápida que sí requirieron de intubación el $37.7 \%$ vivió, a diferencia
Figura 3. Tiempo puerta UTI de acuerdo a los pacientes valorados contra los no valorados por el ERR.

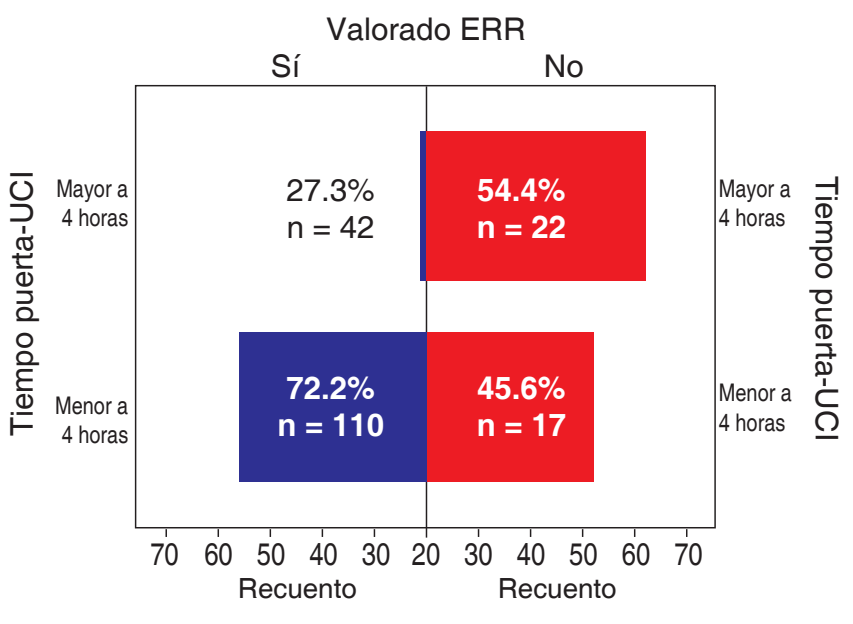

\begin{tabular}{|c|c|c|}
\hline \multicolumn{3}{|c|}{$\begin{array}{l}\text { Cuadro 3. Supervivencia de los pacientes que } \\
\text { requirieron intubación inmediata comparando los } \\
\text { pacientes valorados contra los no valorados por el ERR }\end{array}$} \\
\hline & $\begin{array}{c}\text { Necesidad de } \\
\text { intubación inmediata }\end{array}$ & Supervivencia \\
\hline $\begin{array}{l}\text { Grupo } 1 \\
\mathrm{n}=152 \\
\text { Grupo } 2 \\
\mathrm{n}=39\end{array}$ & $\begin{array}{l}54.5 \% \\
n=83 \\
45.5 \% \\
n=18\end{array}$ & $\begin{array}{c}37.7 \% \\
\mathrm{n}=31 \\
21.2 \% \\
\mathrm{n}=4\end{array}$ \\
\hline
\end{tabular}

de los que no fueron valorados por el equipo de respuesta rápida y requirieron de intubación, sólo el $21.2 \%$ vivió. Se obtuvo una $\mathrm{p}=0.00$ por el método de chi-cuadrada $\left(\chi^{2}\right)$ (Cuadro 3).

\section{Supervivencia de acuerdo a gravedad por NEWS}

Entre los pacientes valorados por el equipo de respuesta rápida (ERR) que obtuvieron una puntuación en la escala de gravedad News menor o igual a 7 y que requirieron de intubación inmediata la supervivencia fue de $42.9 \%$, en contraste con los pacientes que no fueron valorados por el equipo de respuesta rápida (ERR) y que obtuvieron una puntuación en la escala de gravedad News menor o igual a 7 y que sí requirieron de intubación inmediata donde el $35.7 \%$ sobrevivió.

De los pacientes valorados por el equipo de respuesta rápida (ERR) que obtuvieron una puntuación en la escala de gravedad de News mayor a 7 que sí requirieron intubación inmediata el $27.8 \%$ vivió, a diferencia de los paciente no valorados por el equipo de respuesta rápida 
que sí requirieron de intubación inmediata donde el 7\% vivió. Se obtuvo una $p=0.00$ para código de intubación, $p=0.005$ para pacientes valorados por escala de NEWS.

\section{DISCUSIÓN}

Uno de los criterios para analizar la efectividad del ERR COrresponde a la frecuencia de su activación. Con lo anterior, consideramos que el sistema resultó efectivo considerando que se utilizó durante todos los meses estudiados y que, además, la tendencia hacia un incremento en el número de casos atendidos presentó picos en su utilización posterior a la capacitación brindada al personal del hospital. Cabe destacar que la retroalimentación y entrenamiento son de suma importancia para mantener el proyecto activo, ya que situaciones como la rotación de personal, el ingreso de nuevos profesionales de la salud e incluso la apatía del mismo personal, son situaciones que ponen en riesgo la continuidad del ERR. No existe consenso acerca del número de llamadas que se deben realizar por mes. En la literatura se reportan como promedio entre 20 y 30 Ilamadas por cada 1,000 ingresos hospitalarios. ${ }^{17}$ En este estudio se calcularon 7.3 llamadas al ERR por cada 1,000 ingresos hospitalarios.

Por otro lado, analizamos el número de "fallas para rescatar", lo cual se define como el fallecimiento de un paciente posterior a una complicación médica o quirúrgica ${ }^{18}$ durante este periodo, identificando que sólo el $0.05 \%$ de la población que falleció en el Área de Hospitalización presentó datos previos de deterioro sin ser valorados por el ERR hasta presentar paro cardiorrespiratorio. Con lo anterior, no queremos decir que los cuatro pacientes involucrados hubieran sobrevivido al ser ingresados a la Unidad de Cuidados Intensivos, pero sí pudieron haber tenido una mayor oportunidad de recuperación. Cabe mencionar que el considerar estos casos como fallas, es resultado de un análisis por un grupo de expertos especialistas en medicina crítica. No existe un estándar de calidad que indique el número de fallas para rescatar "permitidas" por periodo. Lo importante es asociar estos casos con otros indicadores de calidad como son incidencia de infecciones nosocomiales, enfermedad tromboembólica, marcadores de gravedad, relación enfermera-paciente, etcétera. Así mismo se debe continuar monitorizando y analizando las muertes ocurridas identificando grupos de riesgo y procurando que los casos vayan disminuyendo con el paso del tiempo. Un estudio realizado en Inglaterra de forma retrospectiva incluyó la población de pacientes quirúrgicos durante el periodo de 1997 al 2009 que correspondió a 66,100,672 casos, de los cuales 442,462 fallecieron después de una complicación $(0.7 \%) .{ }^{19}$
El tercer aspecto que analizaremos y que es considerado el de mayor trascendencia en el presente estudio es el resultado de la intervención del equipo de respuesta rápida. Hemos presentado que indiscutiblemente la supervivencia de los pacientes valorados por el ERR es mayor que la de los pacientes no valorados, lo anterior independientemente de si su gravedad es mayor o menor. Además, cabe mencionar que la mortalidad real de este grupo es menor que la esperada de acuerdo al modelo predictor de mortalidad SAPS 3 (26.6\% esperada, 20.3\% real. Índice de mortalidad estandarizado 0.76), lo que confirma que la calidad de la atención en este grupo es adecuada. ${ }^{20}$

Consideramos que varios factores influyen en el buen pronóstico de los pacientes valorados por el ERR. Demostramos que el índice de pacientes que requirieron de intubación inmediata es mayor en aquellos valorados, y que al atender a los pacientes de manera temprana y realizar una intubación oportuna se da una mayor supervivencia. Así mismo, el tiempo puerta-terapia es menor a cuatro horas y esto corresponde con lo reportado en otras publicaciones donde se demuestran mejores resultados con la admisión oportuna y electiva de los pacientes a la Unidad de Cuidados Intensivos, reportando diferencias en la tasa de mortalidad de $36 \%$ en los pacientes ingresados de forma urgente contra $14 \%$ en los pacientes ingresados de forma electiva. ${ }^{21}$

Por otro lado, es de suma importancia considerar que 39 pacientes de 191 ingresados a la Unidad de Cuidados Intensivos (20.4\% de la población) no fueron valorados por el ERR. De esta manera se debe continuar con el entrenamiento del personal hospitalario con el objeto de llegar a la meta de que todos los pacientes ingresados a $\mathrm{UCI}$ provenientes del Área de Hospitalización sean valorados por el ERR ya habiendo mencionado los grandes beneficios que se tienen en tiempo puerta-terapia y supervivencia.

\section{CONCLUSIÓN}

En el Hospital Ángeles Pedregal se documenta que el ERR trabaja con efectividad logrando el reconocimiento temprano del paciente con deterioro clínico, admisión oportuna a UTI y mayor supervivencia.

\section{REFERENCIAS}

1. Davis P, Lay-Yee R, Briant R, Ali W, Scott A, Schug S. Adverse events in New Zealand public hospitals I: Occurrence and impact. N Z Med J. $2002 ; 115$ (1167): U271.

2. Smith AF, Wood J. Can some in-hospital cardio-respiratory arrests be prevented? A prospective survey. Resuscitation. 1998; 37: 133-137.

3. Kause J, Smith G, Prytherch D, Parr M, Flabouris A, Hillman K et al. A comparison of antecedents to cardiac arrests, deaths and emergency intensive care admissions in Australia and New Zealand, and in the 
United Kingdom- the ACADEMIA study. Resuscitation. 2004; 62: 275-282.

4. Schein RM, Hazday N, Pena M, Ruben BH, Sprung CL. Clinical antecedents to in-hospital cardiopulmonary arrest. Chest. 1990; 98: 1388-1392.

5. National Confidential Enquiry into Patient Outcome and Death. An acute problem? London: National Confidential Enquiry into Patient Outcome and Death; 2005.

6. McQuillan P, Pilkington S, Allan A, Taylor B, Short A, Morgan G et al. Confidential inquiry into the quality of care before admission to intensive care. BMJ. 1998; 316: 1853-1858.

7. Intensive Care Society. Guidelines for the introduction of Outreach services. Standards and guidelines. ICS. 2002.

8. Maharaj R, Raffaele I, Wendon J. Rapid response systems: a systematic review and meta-analysis. Crit Care. 2015; 19: 254.

9. Peberdy MA, Cretikos M, Abella BS, DeVita M, Goldhill D, Kloeck $W$ et al. Recommended guidelines for monitoring, reporting, and conducting research on medical emergency team, outreach, and rapid response systems: an Utstein-style scientific statement: a scientific statement from the International Liaison Committee on Resuscitation (American Heart Association, Australian Resuscitation Council, European Resuscitation Council, Heart and Stroke Foundation of Canada, Inter American Heart Foundation, Resuscitation Council of Southern Africa, and the New Zealand Resuscitation Council); the American Heart Association Emergency Cardiovascular Care Committee; the Council on Cardiopulmonary, Perioperative, and Critical Care; and the Interdisciplinary Working Group on Quality of Care and Outcomes Research. Circulation. 2007; 116: 2481-2500.

10. 5 million lives campaign. Getting started kit: rapid response teams. Cambridge, MA: Institute for Healthcare Improvement; 2008.

11. Oglesby KJ, Lesley D, Welch J, Subbe CP. Score to door time, a benchmarking tool for rapid response systems: a pilot multi-centre service evaluation. Crit Care. 2011; 15: R180.
12. National Patient Safety Agency. Safer care for the acutely ill patient: learning from serious incidents. [Internet] 2007. Available in: www. npsa.nhs.uk

13. Echavarría PE, Cerón DU, Esponda PJ, Cabrera JR. Calidad en la atención del paciente crítico. Rev Asoc Mex Med Crity Ter Int. 2012; 26 (1): 42-50.

14. Aguirre SCA, Cerón DUW, Sierra UA. Comparación del rendimiento de 2 modelos predictivos de mortalidad: SAPS 3 vs, APACHE II, en una Unidad de Terapia Intensiva Mexicana. Rev Asoc Mex Med Crit y Ter Int. 2007; 21 (3): 119-124.

15. Vincent JL, de Mendonça A, Cantraine F, Moreno R, Takala J, Suter PM et al. Use of the SOFA score to assess the incidence of organ dysfunction/failure in intensive care units: results of a multicentric, prospective study. Crit Care Med. 1998; 26: 1793-1800.

16. Subbe CP, Kruger M, Rutherford P, Gemmel L. Validation of a modified early warning score in medical admissions. QJM. 2001; 94: 521-526.

17. Ranji SR, Auerbach AD, Hurd CJ, O'Rourke K, Shojania KG. Effects of rapid response systems on clinical outcomes: Systematic review and meta-analysis. J Hosp Med. 2007; 2: 422-432.

18. Silber JH, Romano PS, Rosen AK, Wang Y, Even-Shoshan O, Volpp $K G$. Failure to rescue: comparing definitions to measure quality of care. Med Care. 2007; 45 (10): 918-925.

19. Jones Simon, Bottle Alex, Griffiths Peter. An assessment of "Failure to rescue" derived from routine NHS data as a nursing sensitive patient safety indicator for surgical inpatient care. National Nursin Research Unit. January 2011. [https://www.kcl.ac.uk/nursing/research/nnru/ publications/Reports/Failure-to-Rescue.pdf]

20. Elguea EP, Esponda PJ, Cerón DU, García GM. Segundo lugar Premio «Dr. Mario Shapiro». Calidad de la atención en el cuidado del paciente crítico en una Unidad de Terapia Intensiva mexicana del sector privado. Rev Asoc Mex Med Crit y Ter Int. 2012; XXVI (4): 209-214.

21. Young MP, Gooder VJ, McBride K, James B, Fisher ES. Inpatient transfers to the Intensive Care Unit: delays are associated with increased mortality and morbidity. J Gen Intern Med. 2003; 18: 77. 